\title{
The effects of age on cerebral activations: internally versus externally driven processes
}

\author{
Sien Hu ${ }^{1 *}$, Herta H.-A. Chao ${ }^{2,3}$, Alissa D. Winkler ${ }^{1}$ and Chiang-shan R. Li ${ }^{1,4,5 *}$ \\ Department of Psychiatry, Yale University, New Haven, CT, USA \\ ${ }^{2}$ Department of Internal Medicine, Yale University, New Haven, CT, USA \\ ${ }^{3}$ Department of Internal Medicine, VA Connecticut Healthcare System, West Haven, CT, USA \\ ${ }^{4}$ Department of Neurobiology, Yale University, New Haven, CT, USA \\ ${ }_{5}$ Interdepartmental Neuroscience Program, Yale University, New Haven, CT, USA
}

\section{Edited by:}

Hari S. Sharma, Uppsala University, Sweden

\section{Reviewed by:}

Katsutoshi Furukawa, Tohoku

University, Japan

Eugene A. Kiyatkin, National

Institute on Drug Abuse, USA

\section{*Correspondence:}

Sien Hu, Department of Psychiatry, Connecticut Mental Health Center S108, Yale University, 34 Park

Street, New Haven, CT 06519, USA.

e-mail: sien.hu@yale.edu

Chiang-shan R. Li, Department of Psychiatry, Connecticut Mental Health Center S112, Yale University, 34 Park Street, New Haven,

CT 06519, USA.

e-mail: chiang-shan.li@yale.edu
Numerous studies using functional magnetic resonance imaging (fMRI) have described increased or decreased regional brain activations in older as compared to younger adults. This seeming inconsistency may reflect differences in the psychological constructs examined across studies. We hypothesized that behavioral tasks/contrasts engaging internally and externally driven processes are each associated with age-related decreases and increases, respectively, in cerebral activations. We examined the fMRI data of 103 healthy adults, 18-72 years of age, performing a stop signal task (SST), in which a frequent "go" signal triggered a prepotent response and a less frequent "stop" signal prompted inhibition of this response. Greater internally driven processes lead to stop successes (SS) as compared to stop errors (SE), and to speeding up instead of slowing down in go trials. Conversely, externally driven processes contribute to SE trials, which resulted from habitual, unmonitored responses triggered by the go signal (as compared to SS trials), and involved perceptual and cognitive processes elicited by the stop signal (as compared to go trials). Consistent with our hypothesis, the results showed age-related decreases and increases in cerebral activations each during these respective internally and externally driven processes. These findings further elucidate the influence of age on cognitive functioning and provide an additional perspective to understand the imaging literature of aging.

Keywords: age, aging, inhibitory control, endogenous, exogenous, go/no-go, race model

\section{INTRODUCTION}

Age is associated with changes in cognitive performance (Cabeza, 2002; Park and Reuter-Lorenz, 2009). Numerous functional imaging studies examined the neural bases of such changes and described increased or decreased regional brain activations in older as compared to younger adults. Extant literature reported predominantly decreased cerebral activation when the tasks or psychological contrasts reflect internally driven processes. These internally driven processes direct attention to relevant information as dictated by goals, memory mechanisms such as encoding and recalling, switching of response strategy, anticipation, and shifting of attention as instructed by an endogenous cue (Posner, 1980; Posner and Petersen, 1990; Corbetta and Shulman, 2002; Hahn et al., 2006; Gazzaley and D’Esposito, 2007; Pattyn et al., 2008). For instance, comparing active recall with simple recognition of words, Cabeza et al. (2002) and Johnson et al. (2004) reported age-related decreases in left dorsolateral prefrontal cortical (DLPFC) activation. Thomsen et al. (2004) reported age-related decreases in DLPFC activation when participants were cued endogenously to shift attention to one ear in a dichotic-listening task. When participants maintained eye fixation while covertly attending to a peripheral target, prefrontal cortex and basal ganglia decreased activations in older as compared to younger adults (Solbakk et al., 2008). In a continuous performance task, Braver and Barch (2002) showed agerelated decreases in left DLPFC activation during the delay period. Age-related decreases in cortical activations were also observed in the left DLPFC as well as other frontal and parietal structures during verbal and face encoding, where internally driven processes predominate in these tasks (Grady et al., 2002; Logan et al., 2002; Nyberg et al., 2003; Otsuka et al., 2006).

In contrast, age-related increased activations are frequently observed when the behavioral tasks or contrasts involve processes that are predominantly externally driven, as when attention is drawn to salient or novel stimuli that appear suddenly or unpredictably during target recognition and reflexive eye movements (Posner, 1980; Posner and Petersen, 1990; Corbetta and Shulman, 2002; Hahn et al., 2006; Gazzaley and D'Esposito, 2007; Pattyn et al., 2008). For instance, compared to younger adults, older people demonstrated increased activation during word recognition than recall in the right anterior DLPFC (Cabeza et al., 2002; Jimura and Braver, 2010). In recognition of famous vs. nonfamous people's names, older adults showed greater activation in the left medial PFC, left inferior frontal cortex (IFC), and right hippocampus (Nielson et al., 2006). Bilateral frontal and supplementary eye fields showed age-related increases in activations 
during visually guided saccadic eye movements (Nelles et al., 2009). Older adults also showed increased activation in the presupplementary motor area and bilateral inferior frontal gyri (Zysset et al., 2007), as well as in the left PFC and inferior parietal lobule (IPL) (Mathis et al., 2009), when matching incongruent vs. congruent stimuli.

Overall, these findings suggest that age is associated with decreased and increased cerebral activations each during internally and externally driven processes. We tested this hypothesis by using a large functional magnetic resonance imaging (fMRI) data set collected on the stop signal task (SST). In the SST, participants are required to respond to a go signal and withdraw their response when a stop signal appears. Because go trials are more frequent $(\sim 75 \%)$ than stop trials $(\sim 25 \%)$, the task sets up a prepotent response-pressing a button to the go signal. Thus, successful stopping in the stop trials requires constant monitoring of the stop signal. When participants are engaged in unmonitored responses - as when they are not watching out for the stop signal-in a trial when the stop signal does appear, they are more likely to fail in stopping than when they are monitoring for the stop signal. Thus, stop success (SS) involves greater allocation of attention and response inhibition than stop error (SE) trials (Li et al., 2006, 2008b; Chao et al., 2009; Duann et al., 2009).

Conversely, compared to SS trials, SE trials involve activities related to unmonitored responses and activities that are elicited by the error signals (Li et al., 2008a). Similarly, compared to go success (GS), SE trials share activities of motor execution but involve additional odd-ball (infrequency of stop trials) and error-specific responses. Therefore, a contrast of SE $>$ SS and $\mathrm{SE}>\mathrm{GS}$ engages greater externally driven processes (Ide and $\mathrm{Li}$, 2011a,b; Zhang and Li, 2012). In addition, as we showed previously, participants tend to slow down in their go trial responses with time during the SST. While participants maintained an adequate success rate $(>95 \%)$ in go responses, the mean go reaction time (goRT) increased monotonically across sessions (Chao et al., 2009). This occurs presumably because of a gradual change of response strategy or shift to a more "conservative" mental set. Despite a general trend in slowing down during go responses, participants varied from trial to trial in their goRT. Thus, a go trial in which participants speed up, compared to all previous go trials, potentially represents a distinct scenario where they decide to deviate from the norm in go responses, risking that a stop signal is not imminent. We thus examined whether these go trials, in which they showed a decreased goRT (as compared to the mean goRT of all previous go trials), would actually involve specific regional brain activations, as compared to those go trials that showed an increased goRT (Li et al., 2009; Yan and Li, 2009; Bednarski et al., 2012). Indeed, the results showed a distinct network of cortical and subcortical activations that have previously been implicated in risk taking (Li et al., 2009). Thus, a decision to speed up rather than to slow down after a go trial is internally driven, as participants risk for a no-show of the stop signal in that trial (Li et al., 2009).

Altogether, we can specify four distinct contrasts, two of which each involved predominantly internally and externally driven processes, in the SST. The SST thus offered a unique opportunity to examine the hypothesis with a single behavioral task.

\section{MATERIALS AND METHODS PARTICIPANTS AND BEHAVIORAL TASK}

One hundred and three healthy adults (51 females) with a mean age of 32.5 ( $\mathrm{SD}=12.4$ ) years participated in the study (Table $\mathbf{1}$ ). Participants met the following criteria for recruitment: age 18 and older; right-handed and able to read and write English; no current or history of diagnosis of any Axis I psychiatric or substance (except nicotine) use disorders (First et al., 1995); no current use of psychotropic medications; no significant current medical including neurological, cardiovascular, endocrine, renal, hepatic, or thyroid disorders. Women who were pregnant or breast feeding were not recruited. Participants tested negative for cocaine, amphetamines, opioids, and benzodiazepines prior to fMRI. All participants signed a written consent after they were given a detailed explanation of the study in accordance with a protocol approved by the Yale Human Investigation Committee.

All participants performed a SST (Figure 1; Logan et al., 1984; Li et al., 2006; Chao et al., 2009; Hu and Li, 2011). In the SST, two types of trials, "GO" and "STOP" were randomly presented with an inter-trial-interval (ITI) of $2 \mathrm{~s}$ (the time between the end of the previous trial and the start of the current trial). A fixation dot appeared on the screen to signal the beginning of each trial. The dot was replaced by a circle-the go signal-after a time interval, the "fore-period," which varied from 1 to $5 \mathrm{~s}$ (with uniform distribution). The randomized fore-period minimized anticipation and allowed jittering of events of interest. Participants were instructed to press a button on a hand-held button box using the right index finger when they saw the circle. The circle disappeared at button press or after $1 \mathrm{~s}$ if the participant failed to respond.

In approximately one quarter of the trials, the circle was followed by a "cross" - the stop signal. Participants were instructed to withhold button press when they saw the stop signal. The trial terminated at button press or after $1 \mathrm{~s}$ if the participant successfully inhibited the response. The time between the go and stop signals, the stop signal delay (SSD), started at $200 \mathrm{~ms}$ and varied from one stop trial to the next according to a staircase procedure. If the participant successfully inhibited the response, the SSD increased by $67 \mathrm{~ms}$ in the next stop trial; if the participant failed in inhibiting the response, the SSD decreased by $67 \mathrm{~ms}$. With the staircase procedure we anticipated that participants would succeed in withholding the response half of the time.

Participants were trained briefly on the task before imaging to ensure that they understood the task. They were instructed

Table 1 | The number of subjects broken down by age and gender.

\begin{tabular}{llll}
\hline Age & All & Men & Women \\
\hline $18-19$ & 2 & 1 & 1 \\
$20-29$ & 57 & 24 & 33 \\
$30-39$ & 24 & 15 & 9 \\
$40-49$ & 5 & 3 & 2 \\
$50-59$ & 8 & 5 & 3 \\
$60-69$ & 6 & 4 & 2 \\
$\geq 70$ & 1 & 0 & 1
\end{tabular}




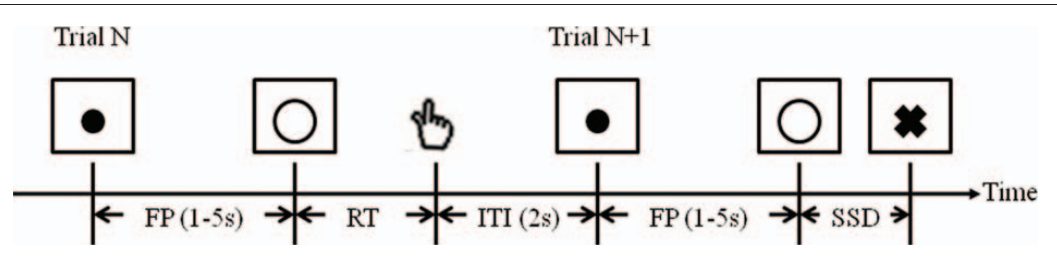

FIGURE 1 | Stop signal paradigm. In GO trials (approximately 75\%, Trial N), participants responded to the go signal (a circle); in STOP trials (approximately $25 \%$, Trial $N+1)$, they had to withhold the response when they saw the stop signal (a cross). In both trials, the go signal appeared after a randomized time interval between 1 and $5 \mathrm{~s}$ (the fore-period or FP, uniform distribution) following the appearance of the fixation point. The stop signal followed the go signal by a time delay - the stop signal delay (SSD). The SSD was updated according to a staircase procedure, whereby it increased or decreased by $67 \mathrm{~ms}$ following a stop success (SS) or stop error (SE) trial, respectively. The inter-trial-interval (ITI) was $2 \mathrm{~s}$. In this diagram, a GO trial was followed by a STOP trial to illustrate the two trial types and the ITI. to quickly press the button when they saw the go signal while keeping in mind that a stop signal might come up in some trials. In the scanner, they completed four sessions of the task, each lasting $10 \mathrm{~min}$, with approximately 100 trials in each session.

\section{BEHAVIORAL DATA ANALYSIS}

A critical SSD was computed for each participant that represented the time delay required for the participant to successfully withhold a response in half of the stop trials (Levitt, 1971). SSDs across stop trials were grouped into runs (sequences of trials), with each run defined as a monotonically increasing or decreasing series. We derived a mid-run estimate by taking the middle SSD of every second run. The critical SSD was computed by taking the mean of all mid-run SSDs. It was reported that, except for experiments with a small number of trials (less than 30), the mid-run estimate was close to the maximum likelihood estimate of $X_{50}$ (50\% SS in the SST, Wetheril et al., 1966). The stop signal reaction time (SSRT) was then computed for each participant by subtracting the critical SSD from the median go trial reaction time (goRT) (Logan et al., 1984).

\section{IMAGING PROTOCOL}

Conventional T1-weighted spin-echo sagittal anatomical images were acquired for slice localization using a 3T scanner (Siemens Trio). Anatomical images of the functional slice locations were next obtained with spin-echo imaging in the axial plan parallel to the AC-PC line with $\mathrm{TR}=300 \mathrm{~ms}$, TE $=2.5 \mathrm{~ms}$, bandwidth $=300 \mathrm{~Hz} /$ pixel, flip angle $=60^{\circ}$, field of view $=220 \times$ $220 \mathrm{~mm}$, matrix $=256 \times 256,32$ slices with slice thickness $=$ $4 \mathrm{~mm}$ and no gap. Functional blood oxygenation level dependent (BOLD) signals were then acquired with a single-shot gradient-echo echo-planar imaging (EPI) sequence. Thirty-two axial slices parallel to the AC-PC line covering the whole brain were acquired with $\mathrm{TR}=2000 \mathrm{~ms}, \mathrm{TE}=25 \mathrm{~ms}$, bandwidth $=$ $2004 \mathrm{~Hz} /$ pixel, flip angle $=85^{\circ}$, field of view $=220 \times 220 \mathrm{~mm}$, matrix $=64 \times 64,32$ slices with slice thickness $=4 \mathrm{~mm}$ and no gap. Three hundred images were acquired in each run for a total of four runs.

\section{SPATIAL PRE-PROCSESING OF BRAIN IMAGES}

Data were analyzed with Statistical Parametric Mapping (SPM8, Wellcome Department of Imaging Neuroscience, University College London, UK). Images from the first five TRs at the beginning of each trial were discarded to enable the signal to achieve steady-state equilibrium between radio frequency pulsing and relaxation. Images of each participant were realigned (motion-corrected) and corrected for slice timing. A mean functional image volume was constructed for each participant for each run from the realigned image volumes. These mean images were co-registered with the high resolution structural image and then segmented for normalization to an MNI (Montreal Neurological Institute) EPI template with affine registration followed by non-linear transformation (Friston et al., 1995a; Ashburner and Friston, 1999). Finally, images were smoothed with a Gaussian kernel of $8 \mathrm{~mm}$ at Full Width at Half Maximum.

\section{GENERAL LINEAR MODEL}

There were four possible trial outcomes: GS, go error (GE), SS, and SE. A statistical analytical design was constructed for each participant using the general linear model (GLM) with the onsets of the go signal from every trial convolved with a canonical hemodynamic response function (HRF) and the temporal derivative of the canonical HRF (Friston et al., 1995b). For GS trials, the go signal onset was parametrically modulated by the reaction time and its temporal derivative; for SS and SE trials, the go signal onset was parametrically modulated by the SSD and its temporal derivative (Li et al., 2006). Realignment parameters in all six dimensions were also entered in the model. Serial autocorrelation of the time series was corrected by a first degree autoregressive or AR (1) model (Friston et al., 2000; Della-Maggiore et al., 2002). The data were high-pass filtered (1/128 Hz cutoff) to remove low-frequency signal drifts. The GLM estimated the component of variance that could be explained by each of the regressors.

A contrast of SS $>$ SE, SE $>$ SS, and SE $>$ GS was made from the first GLM for individual participants. Compared to SE, SS trials involved greater internally driven processes such as attentional monitoring and response inhibition. Compared to SS and GS trials, SE trials involved habitual, unmonitored response to the go signal and error feedbacks, respectively; thus both SE $>$ SS and $\mathrm{SE}>\mathrm{GS}$ involved externally driven processes.

In the second GLM, the GS trials were divided into those that are followed by a GS (pG), GE (pGE), SS (pSS), and SE (pSE) trial, with $\mathrm{pG}$ trials further divided into those that decreased in goRT (pGd) and increased in goRT (pGi), as compared to the mean of 
all previous GS trials (Li et al., 2009). We contrasted for individual participants pGd > pGi (post-go speeding > post-go slowing), a process that is internally driven and involves a decision to risk that an upcoming trial will not be a stop trial.

Altogether, we thus had four contrasts, two of which (SS $>$ SE and $\mathrm{pGd}>\mathrm{pGi}$ ) were predominately internally driven and the other two (SE > SS and SE > GS) externally driven. In group level analyzes, we ran a one-sample $t$-test to identify regions of interest (ROI) for each of the four contrasts. We then performed a multiple regression against age and gender for each of the four contrasts with small volume correction (SVC) for the ROIs. By regressing only voxels of the ROIs identified for each contrast, we ensured that the correlations with age reflect an association specific to the psychological construct of interest. For instance, a positive correlation of age with the contrast SS $>$ SE would identify only voxels that showed greater activation during SS as compared to SE trials, reflecting attentional monitoring and response inhibition. Without restricting the regression to the ROIs, a positive correlation of age with SS $>$ SE would also identify voxels that correlated negatively with age for $\mathrm{SE}>\mathrm{SS}$.

We used MarsBaR (Brett et al., 2002) to derive for each participant the effect size of activity change for the ROIs identified in each contrast for the regression with age.

\section{RESULTS}

\section{BEHAVIORAL PERFORMANCE}

Table 2 summarized behavioral performance on the SST. On average, participants made errors in about half of the stop trials, suggesting that their overall performance was adequately tracked by the staircase procedure. We correlated behavioral performance with age (Figure 2). Pearson's correlations showed that the median goRT, SSRT, and the GS rate were significantly correlated with age (Median goRT, $r=0.2606, p=0.0078$; SSRT, $r=0.3703, p=0.0001$; GS\%, $r=-0.3218, p=0.0009)$, and the SS rate showed a trend toward significant correlation with age (SS\%, $r=0.2046, p=0.0382$ ), corrected for multiple comparisons $(p<0.0083)$. The fore-period effect $(r=-0.1067, p=$ $0.2833)$ and the post-error slowing $(r=-0.0287, p=0.7739)$ were not correlated with age.

Table 2 | Behavioral performance on the stop signal task $(n=103)$.

\begin{tabular}{lll}
\hline & Range & Mean \pm Standard deviation \\
\hline Median goRT & $315-784 \mathrm{~ms}$ & $589 \pm 101 \mathrm{~ms}$ \\
Mean goRT & $335-782 \mathrm{~ms}$ & $595 \pm 94 \mathrm{~ms}$ \\
SSRT & $98-287 \mathrm{~ms}$ & $204 \pm 34 \mathrm{~ms}$ \\
GS\% & $84.5-100 \%$ & $95.8 \pm 3.6 \%$ \\
SS\% & $44.9-57.8 \%$ & $50.5 \pm 2.3 \%$ \\
FP effect (effect size) & $-0.96-6.04$ & $2.38 \pm 1.64$ \\
PES (effect size) & $-2.41-7.59$ & $2.04 \pm 1.71$
\end{tabular}

Note: goRT: go trial reaction time; SSRT: stop signal reaction time; GS\%: percentage of go success trials in all go trials; SS\%: percentage of stop success trials in all stop trials; FP: fore-period; PES: post-error slowing. Effect sizes rather than mean changes were computed for FP effect and PES to account for slight variation in the number of trials between participants.

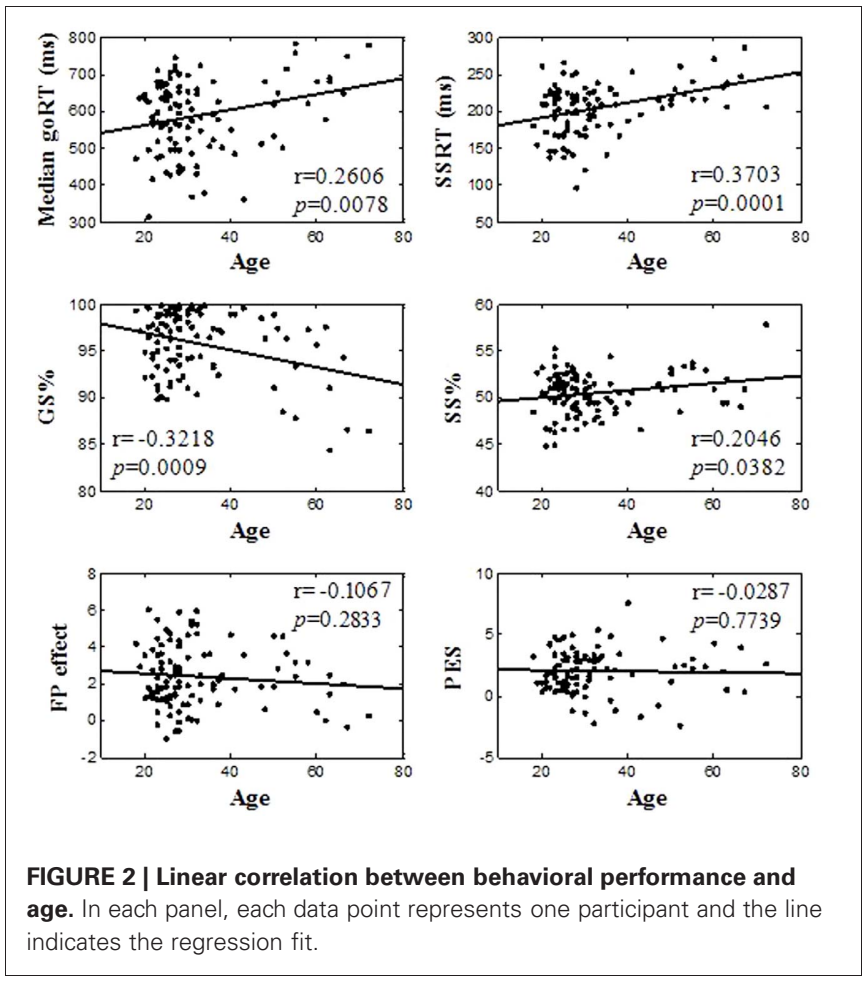

THE EFFECTS OF AGE ON REGIONAL BRAIN ACTIVATIONS

We first examined the influence of gender by including both age and gender in the regressions. The results showed no regional activations associated with gender at $p<0.001$ uncorrected. We, therefore, reported age-related activations by combining men and women.

The regressions showed age-related decreases and increases in activations for internally and externally driven processes, respectively (Table 3 ). Figure 3 shows activations in each contrast overlaid on a T1 structural image using MRIcroN (Rorden and Brett, 2000). In SS > SE, age was associated with decreased activation in the left orbitofrontal cortex (OFC) and dorsal lateral prefrontal cortex (DLPFC) at $p<0.05$, corrected for family wise errors (FWE) of multiple comparisons. In pGd > pGi, age was associated with decreased activations in the left inferior frontal cortex (IFC) at $p<0.05$, corrected for FWE. For both contrasts, no brain regions showed age-associated increases in activation even when queried against larger masks derived with an uncorrected $p<0.05$.

In $\mathrm{SE}>\mathrm{SS}$, age was associated with increased activation in bilateral primary motor cortices (PMC), postcentral gyri, supplementary motor area (SMA), bilateral superior temporal gyrus (STG), and the right IFC/insula. In SE > GS, age was associated with increased activation in bilateral PMC, the right inferior parietal lobule (IPL), and the left IFC/insula. Likewise, for both $\mathrm{SE}>\mathrm{SS}$ and $\mathrm{SE}>\mathrm{GS}$, no brain regions showed age-associated decreases in activation even when queried against larger masks derived with an uncorrected $p<0.05$.

We extracted the effect sizes of activation for each contrast to correlate with age. The effect size of SS $>$ SE correlates inversely with age in the left OFC $(r=-0.3274, p=0.0007)$ and DLPFC 
Table 3 | (A) Age-correlated regional brain activations during internally driven processes. (B) Age-correlated regional brain activations during externally driven processes.

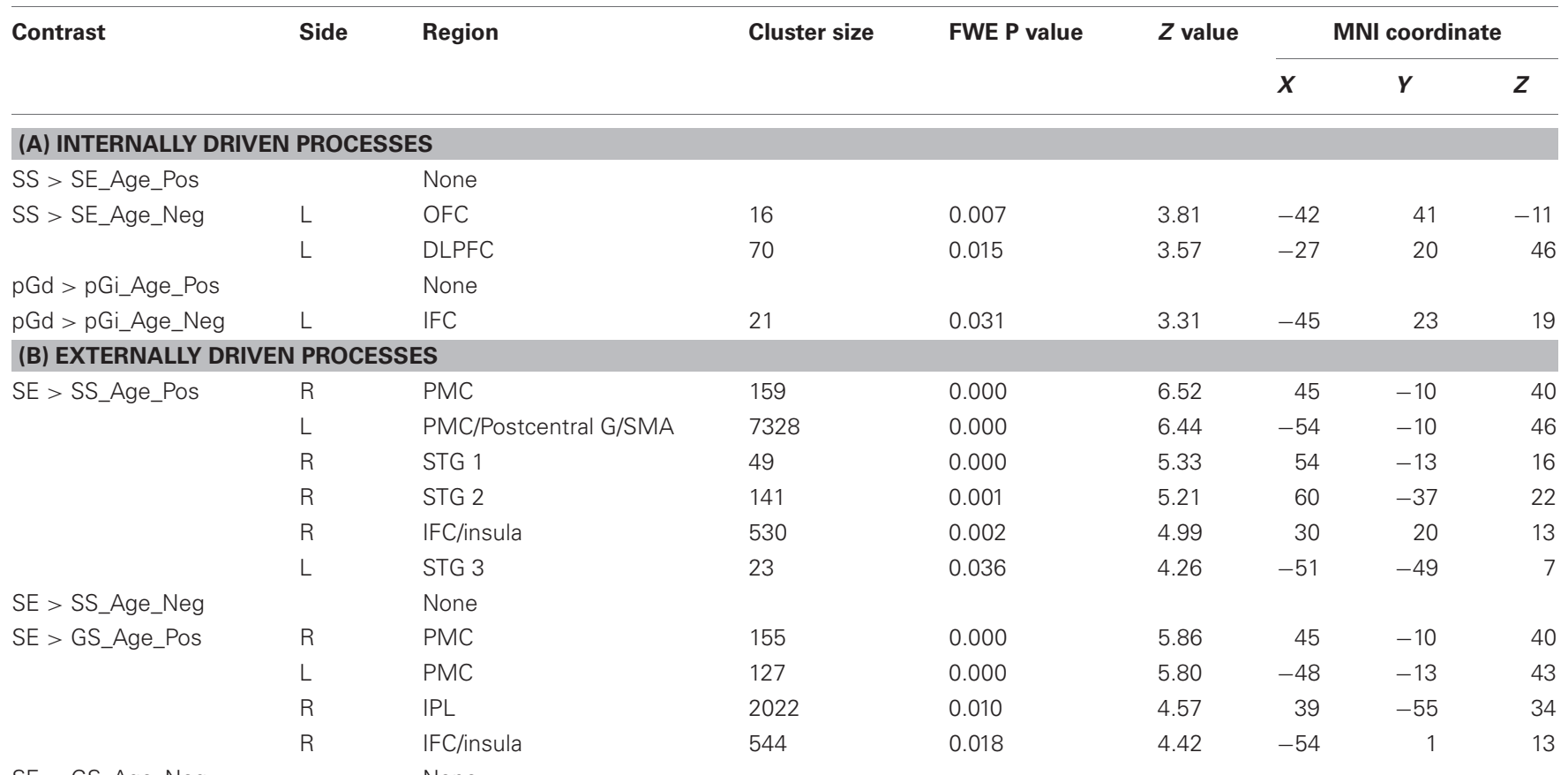

SE > GS_Age_Neg

None

Note: Age_Pos: positive correlation with age; Age_Neg: negative correlation with age. OFC: orbitofrontal cortex; DLPFC: dorsal lateral prefrontal cortex; IFC: inferior frontal cortex; PMC: primary motor cortex; SMA: supplementary motor area; STG: superior temporal gyrus; IPL: inferior parietal lobule; G: gyrus.

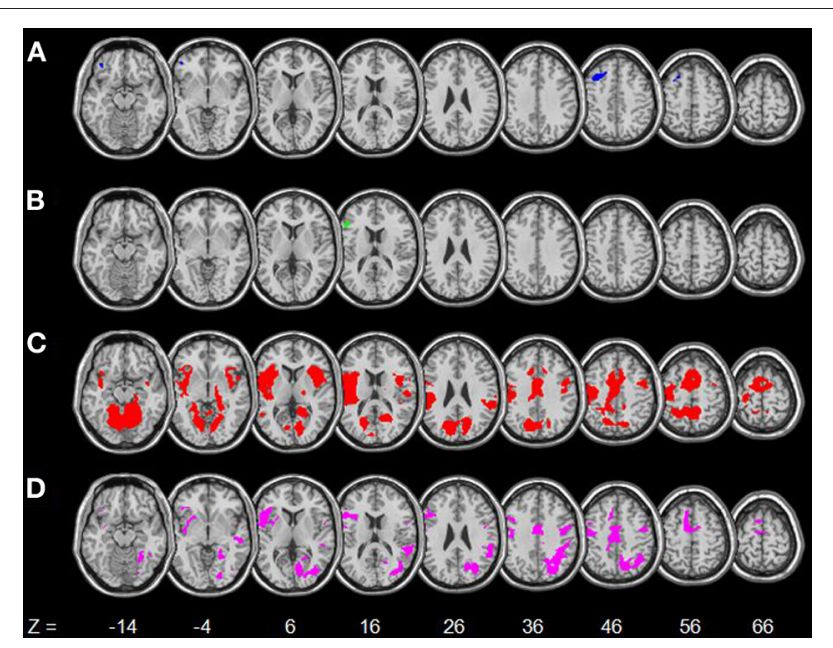

FIGURE 3 | Age-related changes in regional brain activations. (A) Blue: age-related decreased activations in SS > SE; (B) Green: age-related decreased activations in $\mathrm{pGd}>\mathrm{pGi}$; (C) Red: age-related increased activations in SE > SS; (D) Magenta: age-related increased activations in SE > GS. Numbers at the bottom indicate the positions in the $z$-axis in the $\mathrm{MNI}$ coordinate. Neurological orientation: right = right.

$(r=-0.3094, p=0.0015)$. Figure 4A highlights the correlation for the left OFC of SS $>$ SE. The effect size of $\mathrm{pGd}>\mathrm{pGi}$ also correlates inversely with age in the left IFC $(r=-0.2216$, $p=0.0327$, Figure 4B). Similarly, the effect sizes of all SE $>$ SS regions $(r \mathrm{MEAN}=0.3933, \mathrm{pMEAN}=0.0000)$ and $\mathrm{SE}>\mathrm{GS}$
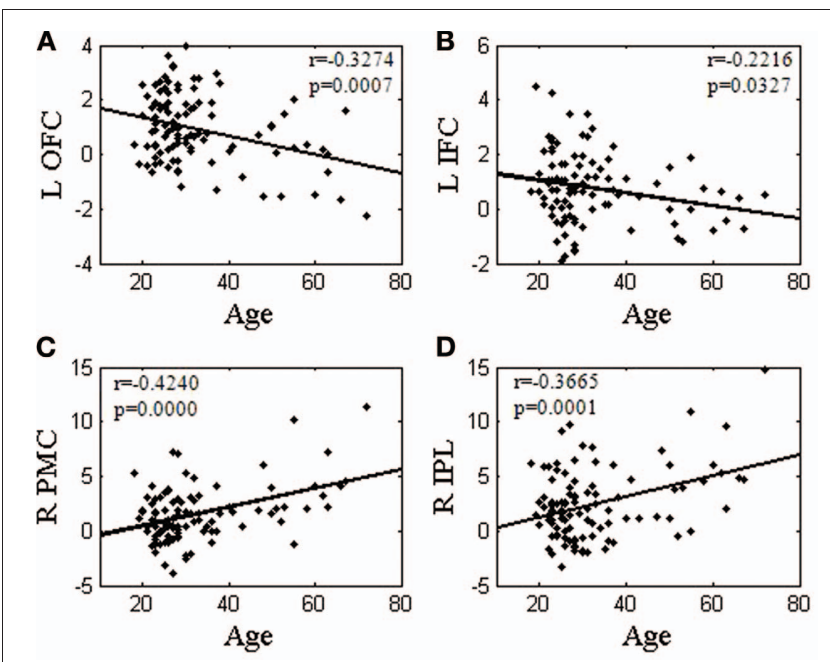

FIGURE 4 | Linear correlation between effect sizes of regional brain activations and age. Regional brain activations of internally driven processes (A: SS > SE; and $\mathbf{B}$ : pGd > pGi) were negatively correlated with age. Regional brain activations of externally driven processes (C: $S E>$ SS; and D: SE > GS) were positively correlated with age. L: left; R: right: OFC: orbital frontal cortex; IFC: inferior frontal cortex; PMC: primary motor cortex; IPL: inferior parietal lobule.

regions $(r \mathrm{MEAN}=0.3602, p \mathrm{MEAN}=0.0001)$ correlate positively with age. Figure 4C highlights the correlation of the right PMC in $\mathrm{SE}>\mathrm{SS}(r=0.4240, p=0.0000)$, and Figure 4D of the right IPL in SE $>$ GS $(r=0.3665, p=0.0001)$. 
To take into account variation in behavioral performance, in a second analysis we included both age and SSRT as regressors. The patterns of age-related activations were very similar to those of the first analysis with age as the only regressor. In particular, $\mathrm{SS}>\mathrm{SE}$ revealed age-related decreases in left OFC $(x=-42, y=41$, $z=-11, Z=3.84, p_{F W E}=0.006$, cluster size $=16$ voxels $)$ and DLPFC $\left(x=-27, y=20, z=-46, Z=3.60, p_{F W E}=0.014\right.$, cluster size $=53$ voxels) activation; $\mathrm{pGd}>\mathrm{pGi}$ was associated with age-related decreases in left IFC $(x=-45, y=23, z=19$, $Z=2.96$, $p_{F W E}=0.088$, cluster size $=21$ voxels) activation. $\mathrm{SE}>\mathrm{SS}$ showed age-related increases in right PMC $(x=48$, $y=-7, z=40, Z=6.02, p_{F W E}=0.000$, cluster size $=163$ voxels), left PMC/postcentral gyri/SMA $(x=-54, y=-10, z=$ $46, Z=5.81, p_{F W E}=0.000$, cluster size $=5847$ voxels $)$, right STG $\left(x=54, y=-13, z=16, Z=5.20\right.$, $p_{F W E}=0.001$, cluster size $=49$ voxels), and right IFC/insula $(x=57, y=2, z=$ $13, Z=4.51, p_{F W E}=0.013$, cluster size $=476$ voxels $)$ activation; SE > GS showed age-related increases in bilateral PMC (left: $x=-48, y=-16, z=43, Z=5.41$, $p_{F W E}=0.000$, cluster size $=783$ voxels; right: $x=42, y=-10, z=40, Z=5.31$, $p_{F W E}=0.000$, cluster size $=174$ voxels $)$, right IPL $(x=39$, $y=-55, z=34, Z=4.41, p_{F W E}=0.019$, cluster size $=1737$ voxels), and left IFC/insula $(x=-54, y=-46, z=4, Z=4.03$, $p_{F W E}=0.080$, cluster size $=1052$ voxels $)$ activation.

To further investigate the influence of performance on agerelated activations, we compared 32 younger ( $27.3 \pm 4.5$ years) and 13 older ( $57.1 \pm 6.0$ years) adults matched in SSRT (young: $229 \pm 13 \mathrm{~ms}$; old: $238 \pm 23 \mathrm{~ms}, t=1.671, p=0.102$ ) for each of the four contrasts using two-sample $t$ tests. The results showed that, when queried against a mask of SS $>$ SE at $p<0.001$, uncorrected, a cluster in the left OFC $(x=-48, y=41, z=-5$, $Z=3.72$, $p_{F W E}=0.024$, cluster size $=73$ voxels $)$ showed less activation and no brain regions increased in activation in the older as compared to younger adults.

\section{DISCUSSION}

The current findings support the hypothesis that age is associated with decreased and increased cerebral activations each during internally and externally driven processes. This unique pattern of age-related changes in regional brain activations is observed whether variation in behavioral performance is taken into account or not.

Behaviorally, age is associated with increased goRT and SSRT, consistent with previous research (Bedard et al., 2002; Rush et al., 2006; Gamboz et al., 2009). While increased goRT may reflect declined speed in information processing (Park et al., 2001; Kray et al., 2002; Buckner, 2004; Li et al., 2009; Hammerer et al., 2010) or a more conservative strategy (Reuter-Lorenz and Lustig, 2005; Reuter-Lorenz and Cappell, 2008), increased SSRT suggests age-related changes in cognitive functioning. A recent overview indicated that age-associated deficits in executive functions did not appear to go beyond the variance accounted for by speed-of-processing and working memory, except in aspects of cognitive control that required divided attention (Verhaeghen, 2011). The SST demanded participants to attend to both the go and stop signals so that they could respond quickly and be able to withhold this response when the need arose. This dual requirement may thus elicit age-related changes in response inhibition, a function central to cognitive control. Altogether, these behavioral findings provide evidence validating the use of the SST in studying age-related changes in cerebral activations.

Activations of the left OFC and DLPFC in SS as compared to SE trials, a contrast involving attentional and inhibitory control (Li et al., 2006), were inversely correlated with age. This finding is consistent with a recent study that also showed a negative correlation between age and prefrontal activations during inhibitory control in the SST, in a younger population (Cohen et al., 2010). Activations of the IFC during post-go speeding as compared to post-go slowing trials also were inversely correlated with age. Conversely, activations in many areas, including the bilateral IFC, PMC, postcentral gyri, and superior temporal gyri (STG), in SE as compared to SS or in SE as compared to GS trials were positively correlated with age. Further, no brain regions showed a positive correlation with age during SS $>$ SE or post-go speeding $>$ postgo slowing, or a negative correlation with age during $\mathrm{SE}>\mathrm{SS}$ or SE > GS. These results support the hypothesis that age is related to decreased regional activations during internally driven processes and increased activations during externally driven processes.

This differential pattern of regional activations appears to be consistent with the top-down and bottom-up attention cortical circuits as proposed by Corbetta and Shulman (2002). These results are also in accord with the cortical networks of endogenous and exogenous control during saccadic eye movements. Specifically, Mort et al. (2003) reported predominantly frontal and parietal activations, respectively, each during voluntary (internally driven) and reflexive (externally driven) saccades. Thus, age-related decreases of activation in the dorsal frontal areas and increases of activation in the ventral frontal, temporal, and occipital visual cortices are consistent with this functional dichotomy of cortical systems.

In addition, these results are in line with the pattern of regional brain activations associated with dual mechanisms of control, as proposed by Braver and colleagues (Braver et al., 2007). Proactive control maintains goal-related information before target appearance and reactive control operates at a later stage for conflict detection and resolution. Using a continuous performance task, Braver et al. (2009) reported age-related decreases of sustained activity in the lateral PFC and increases of transient activity in the same prefrontal as well as posterior parietal regions. It was suggested that decreased and increased cortical activation reflect, respectively, inferiority of proactive control and greater reliance on reactive control in older adults (Paxton et al., 2008; Braver et al., 2009; Jimura and Braver, 2010).

In an electrophysiological study of continuous performance task, Hammerer et al. (2010) reported a greater P3 response to cue than non-cue stimulus in younger adults but a greater P3 response to non-cue than cue stimulus in older adults. Their finding suggested that younger adults allocate attention to the more frequent cue stimuli while older adults are more attracted by the infrequent and unexpected stimuli. These results are consistent with our findings of age-related decreased activation in attentional regions during internally driven processes, and age-related 
increased activation in motor-sensory related areas during externally driven processes.

Most behavioral tasks engage both internally and externally driven processes (Corbetta and Shulman, 2002). For example, behavioral paradigms that are widely used to study cognitive control-Stroop, go/no-go, and flanker tasks-involve both intentional suppression of a prepotent response (internally driven) and processing of odd-ball effects (externally driven) during incongruent, as compared to congruent, trials. Thus, by comparing incongruent and congruent trials in the Stroop task, previous studies have reported age-related decreases in bilateral DLPFC (Milham et al., 2002) and left IFC (Langenecker et al., 2004) activations, as well as age-related increases in visual area activations (Milham et al., 2002). Similarly, in a go/no-go task, Nielson et al. (2002) observed age-related decreased activation of the right IFC and fusiform gyrus along with increased activation of the left DLPFC and IFC during "no-go" responses as compared to resting (see also Langenecker and Nielson, 2003). In a flanker task, by comparing incongruent and congruent trials, Colcombe et al. (2005) reported age-related increases in activation of bilateral DLPFC, left IFC, and anterior cingulate cortex/SMA, as well as decreases in activation of the left MFG. Likewise, during verb generation, Persson et al. (2004) found age-related decreases in the left IFC and increases in right IFC activation. In recalling a six letter vs. one letter stimulus, which differed both in perceptual processing and memory load, older adults showed less activation in the right rostral PFC and IFC but greater activation in the right DLPFC (Rypma et al., 2001). Sometimes, the very same brain region increased in activation in one study but decreased in others for identical contrasts. For instance, in comparing incongruent with congruent trials, Milham et al. (2002) reported age-related decreases while Colcombe et al. (2005) reported increases in bilateral DLPFC activation. We speculate that this discrepancy reflected the different extents to which internally vs. externally driven processes were involved across studies.

Past research attributed decreased cerebral activations along with impaired behavioral performance to aging of the brain, and posited compensatory mechanisms to account for age-related increases in regional brain activations (Park et al., 2001; Buckner, 2004; Park and Reuter-Lorenz, 2009). The Compensation-Related Utilization of Neural Circuits Hypothesis (CRUNCH) theorized that overactivation in older adults reflects a compensatory mechanism: as task difficulty increases, older adults fail to continue to increase activation (Reuter-Lorenz and Cappell, 2008). In addition to task difficulty, the interpretation of compensatory mechanisms should take into account behavioral performance, as suggested by Nagel and colleagues (Nagel et al., 2009, 2011). Using a working memory task with different levels of memory load, Nagel et al. reported different patterns of dose-response functions between high and low performers in young as well as in old adults. As memory load increased, young-high performers showed a positive linear increment of activation, and old-low performers showed a quadratic pattern of activation, while young-low performers and old-high performers showed a similar, mixed pattern. The fact that old-high performers presented a youth-like pattern suggested a compensatory mechanism, especially in low load conditions, consistent with the CRUNCH. On the other hand, compared to old-high performers, old-low performers did not sufficiently recruit task-related brain regions even in low load conditions, suggesting a cognitive/neurological impairment associated with age. Nagel and colleagues' research highlighted the impact of heterogeneous neural processes associated with aging on the interpretation of age-related differential patterns and emphasized the integration of imaging results and behavioral performance.

With the reminder from Nagel and colleagues' work, we regressed BOLD contrasts against age both with and without SSRT as a covariate and found that, when behavioral performance was accounted for, age was associated with similar patterns of cerebral activations. Moreover, compared to a group of younger adults who were matched in SSRT, older adults showed similar decreases in OFC activations during SS as compared to SE trials. In no case were increased activations observed in older as compared to younger adults. While these results support decreased cerebral activations during an internally driven process, they do not rule out the possibility of a compensatory mechanism in aging; older adults' performance may have reached ceiling and thus failed to demonstrate compensatory increases in activations (Park et al., 2001; Buckner, 2004; Nagel et al., 2009, 2011; Park and Reuter-Lorenz, 2009).

On the other hand, the current results of increased activation during the externally driven process in SE > SS and SE > GS could reflect a compensatory mechanism in older adults, because post-error slowing, as an index of error processing, did not vary with age. However, we did not observe increased bilateral activation or reduction of hemispheric asymmetry in activation, in association with age (Cabeza, 2002). The current results also did not show accompanying age-related decreases in activations of other brain regions (e.g., Grady et al., 2003) or age-related increases in activations of non-task areas, a dedifferentiation or over-recruitment, to compensate for performance (Park et al., 2004), even when the results were examined at $p=0.05$ uncorrected. Taken together, evidence is mixed regarding the compatibility of the current results with compensatory processes in aging.

A limitation in this study is the small number of participants in the highest age range. Only seven out of 103 people were over 60 years of age. Thus, it remains to be seen whether the pattern of age-related effects will follow the same trend for an elderly population.

In conclusion, the current study investigated how age influences regional brain activations in a SST. We observed agerelated decreases in cerebral activations during internally driven processes and increases in activations during externally driven processes, in support of a functional dichotomy of age-related changes in cerebral activities during cognitive challenges.

\section{ACKNOWLEDGMENTS}

This study was supported by NIH grants K02DA026990 (Li), R03CA138121 (Chao), the William O. Seery Foundation (Chao), and a Yale Cancer Center translational pilot grant (Chao). We thank Dr. Jaime Ide, Dr. Sheng Zhang, Olivia Hendrick, Sarah Bednarski, and Emily Erdman for their many helpful discussions. 


\section{REFERENCES}

Ashburner, J., and Friston, K. J. (1999). Nonlinear spatial normalization using basis functions. Hum. Brain Mapp. 7, 254-266.

Bedard, A. C., Nichols, S., Barbosa, J. A., Schachar, R., Logan, G. D., and Tannock, R. (2002). The development of selective inhibitory control across the life span. Dev. Neuropsychol. 21, 93-111.

Bednarski, S. R., Erdman, E., Luo, X., Zhang, S., Hu, S., and Li, C. S. R. (2012). Neural processes of an indirect analogue of risk taking in young non-dependent adult alcohol drinkers: an fMRI study of the stop signal task. Alcohol. Clin. Exp. Res. doi: 10.1111/j.15300277.2011.01672.x. [Epub ahead of print]

Braver, T. S., and Barch, D. M. (2002). A theory of cognitive control, aging cognition, and neuromodulation. Neurosci. Biobehav. Rev. 26, 809-817.

Braver, T. S., Gray, J. R., and Burgess, G. C. (2007). "Explaining the many varieties of working memory variation: dual mechanisms of cognitive control," in Variation in Working Memory, eds A. R. A. Conway, C. Jarrold, M. J. Kane, A. Miyake, and J. N. Towse (Oxford: Oxford University Press), 76-106.

Braver, T. S., Paxton, J. L., Locke, H. S., and Barch, D. M. (2009). Flexible neural mechanisms of cognitive control within human prefrontal cortex. Proc. Natl. Acad. Sci. U.S.A. 106, 7351-7356.

Brett, M., Johnsrude, I. S., and Owen, A. M. (2002). The problem of functional localization in the human brain. Nat. Rev. Neurosci. 3, 243-249.

Buckner, R. L. (2004). Memory and executive function in aging and $\mathrm{AD}$ : multiple factors that cause decline and reserve factors that compensate. Neuron 44, 195-208.

Cabeza, R. (2002). Hemispheric asymmetry reduction in older adults: the HAROLD model. Psychol. Aging 17, 85-100.

Cabeza, R., Anderson, N. D., Locantore, J. K., and McIntosh, A. R. (2002). Aging gracefully: compensatory brain activity in high-performing older adults. Neuroimage 17, 1394-1402.

Chao, H. H., Luo, X., Chang, J. L., and Li, C. S. (2009). Activation of the pre-supplementary motor area but not inferior prefrontal cortex in association with short stop signal reaction time-an intrasubject analysis. BMC Neurosci. 10,75
Cohen, J. R., Asarnow, R. F., Sabb, F. W., Bilder, R. M., Bookheimer, S. Y., Knowlton, B. J., and Poldrack, R. A. (2010). Decoding developmental differences and individual variability in response inhibition through predictive analyses across individuals. Front. Hum. Neurosci. 4:47. doi: 10.3389/fnhum.2010.00047

Colcombe, S. J., Kramer, A. F., Erickson, K. I., and Scalf, P. (2005). The implications of cortical recruitment and brain morphology for individual differences in inhibitory function in aging humans. Psychol. Aging 20, 363-375.

Corbetta, M., and Shulman, G. L. (2002). Control of goal-directed and stimulus-driven attention in the brain. Nat. Rev. Neurosci. 3, 201-215.

Della-Maggiore, V., Chan, W., PeresNeto, P. R., and McIntosh, A. R. (2002). An empirical comparison of SPM preprocessing parameters to the analysis of fMRI data. Neuroimage 17, 19-28.

Duann, J. R., Ide, J. S., Luo, X., and Li, C. S. (2009). Functional connectivity delineates distinct roles of the inferior frontal cortex and presupplementary motor area in stop signal inhibition. J. Neurosci. 29, 10171-10179.

First, M., Spitzer, R., and Williams, J. M. G. (1995). Structured Clinical Interview for DSM-IV (SCID). Washington, DC: American Psychiatric Association.

Friston, K., Holmes, A. P., Worsley, K. J., Poline, J. B., Frith, C. D., and Frackowiak, R. (1995a). Statistical parametric maps in functional imaging: a general linear approach. Hum. Brain Mapp. 2, 189-210.

Friston, K. J., Ashburner, J., Frith, C. D., Poline, J. B., Heather, J. D., and Frackowiak, R. S. J. (1995b). Spatial registration and normalization of images. Hum. Brain Mapp. 3, 165-189.

Friston, K. J., Josephs, O., Zarahn, E., Holmes, A. P., Rouquette, S., and Poline, J. (2000). To smooth or not to smooth? Bias and efficiency in AMRI time-series analysis. Neuroimage 12, 196-208.

Gamboz, N., Borella, E., and Brandimonte, M. A. (2009). The role of switching, inhibition and working memory in older adults' performance in the wisconsin card sorting test. Neuropsychol. Dev. Cogn. B Aging Neuropsychol. Cogn. 16, 260-284.

Gazzaley, A., and D'Esposito, M. (2007). Top-down modulation and normal aging. Ann. N.Y. Acad. Sci. 1097, 67-83.
Grady, C. L., Bernstein, L. J., Beig, S., and Siegenthaler, A. L. (2002). The effects of encoding task on age-related differences in the functional neuroanatomy of face memory. Psychol. Aging 17, 7-23.

Grady, C. L., McIntosh, A. R., and Craik, F. I. M. (2003). Age-related differences in the functional connectivity of the hippocampus during memory encoding. Hippocampus 13, 572-586.

Hahn, B., Ross, T. J., and Stein, E. A. (2006). Neuroanatomical dissociation between bottom-up and top-down processes of visuospatia selective attention. Neuroimage 32, 842-853.

Hammerer, D., Li, S. C., Muller, V., and Lindenberger, U. (2010). An electrophysiological study of response conflict processing across the lifespan: assessing the roles of conflict monitoring, cue utilization, response anticipation, and response suppression. Neuropsychologia 48 3305-3316.

Hu, S., and Li, C. S. R. (2011). Neura processes of preparatory control for stop signal inhibition. Hum. Brain Mapp. doi: 10.1002/hbm.21399. [Epub ahead of print].

Ide, J. S., and Li, C. S. R. (2011a). A cerebellar thalamic cortical circuit for error-related cognitive control. Neuroimage 54, 455-464.

Ide, J. S., and Li, C. S. R. (2011b) Error-related functional connectivity of the habenula in humans. Front. Hum. Neurosci. 5:25. doi 10.3389/fnhum.2011.00025

Jimura, K., and Braver, T. S. (2010). Age-related shifts in brain activity dynamics during task switching. Cereb. Cortex 20, 1420-1431.

Johnson, M. K., Mitchell, K. J., Raye, C. L., and Greene, E. J. (2004). An agerelated deficit in prefrontal cortical function associated with refreshing information. Psychol. Sci. 15 127-132.

Kray, J., Li, K. Z., and Lindenberger, U. (2002). Age-related changes in task-switching components: the role of task uncertainty. Brain $\operatorname{Cog} n$. 49, 363-381.

Langenecker, S. A., and Nielson, K. A. (2003). Frontal recruitment during response inhibition in older adults replicated with fMRI. Neuroimage 20, 1384-1392.

Langenecker, S. A., Nielson, K. A., and Rao, S. M. (2004). fMRI of healthy older adults during Stroop interference. Neuroimage 21, 192-200.

Levitt, H. (1971). Transformed up down methods in psychoacoustics. J. Acoust. Soc. Am. 49, 467-477.
Li, C. S. R., Chao, H. H. A., and Lee, T. W. (2009). Neural correlates of speeded as compared with delayed responses in a stop signal task: an indirect analog of risk taking and association with an anxiety trait. Cereb. Cortex 19, 839-848.

Li, C. S. R., Huang, C., Constable, R. T., and Sinha, R. (2006). Imaging response inhibition in a stop-signal task: neural correlates independent of signal monitoring and postresponse processing. J. Neurosci. 26 , 186-192.

Li, C. S. R., Yan, P., Chao, H. H. A., Sinha, R., Paliwal, P., Constable, R. T., Zhang, S., and Lee, T. W. (2008a). Error-specific medial cortical and subcortical activity during the stop signal task: a functional magnetic resonance imaging study. Neuroscience 155, 1142-1151.

Li, C. S. R., Yan, P., Sinha, R., and Lee, T. W. (2008b). Subcortical processes of motor response inhibition during a stop signal task. Neuroimage 41, 1352-1363.

Logan, G. D., Cowan, W. B., and Davis, K. A. (1984). On the ability to inhibit simple and choice reaction-time responses - a model and a method. J. Exp. Psychol. Hum. Percept. Perform. 10, 276-291.

Logan, J. M., Sanders, A. L., Snyder, A. Z., Morris, J. C., and Buckner, R. L. (2002). Under-recruitment and nonselective recruitment: dissociable neural mechanisms associated with aging. Neuron 33, 827-840.

Mathis, A., Schunck, T., Erb, G., Namer, I. J., and Luthringer, R. (2009). The effect of aging on the inhibitory function in middle-aged subjects: a functional MRI study coupled with a color-matched Stroop task. Int. J. Geriatr. Psychiatry 24, 1062-1071.

Milham, M. P., Erickson, K. I., Banich, M. T., Kramer, A. F., Webb, A., Wszalek, T., and Cohen, N. J. (2002). Attentional control in the aging brain: insights from an fMRI study of the stroop task. Brain Cogn 49, 277-296.

Mort, D. J., Perry, R. J., Mannan, S. K., Hodgson, T. L., Anderson, E., Quest, R., McRobbie, D., McBride, A., Husain, M., and Kennard, C. (2003). Differential cortical activation during voluntary and reflexive saccades in man. Neuroimage 18 231-246.

Nagel, I. E., Preuschhof, C., Li, S. C., Nyberg, L., Backman, L., Lindenberger, U., and Heekeren, H. R. (2009). Performance level modulates adult age differences in brain activation during spatial working memory. Proc. Natl. Acad. Sci. U.S.A. 106, 22552-22557. 
Nagel, I. E., Preuschhof, C., Li, S. C., Nyberg, L., Backman, L., Lindenberger, U., and Heekeren, H. R. (2011). Load modulation of BOLD response and connectivity predicts working memory performance in younger and older adults. J. Cogn. Neurosci. 23, 2030-2045.

Nelles, G., de Greiff, A., Pscherer, A., and Esser, J. (2009). Age-related differences of saccade induced cortical activation. Neurosci. Lett. 458, 15-18.

Nielson, K. A., Douville, K. L., Seidenberg, M., Woodard, J. L., Miller, S. K., Franczak, M., Antuono, P., and Rao, S. M. (2006). Age-related functional recruitment for famous name recognition: an event-related fMRI study. Neurobiol. Aging 27, 1494-1504.

Nielson, K. A., Langenecker, S. A., and Garavan, H. (2002). Differences in the functional neuroanatomy of inhibitory control across the adult life span. Psychol. Aging 17, 56-71.

Nyberg, L., Sandblom, J., Jones, S., Neely, A. S., Petersson, K. M., Ingvar, M., and Backman, L. (2003). Neural correlates of training-related memory improvement in adulthood and aging. Proc. Natl. Acad. Sci. U.S.A. 100, 13728-13733.

Otsuka, Y., Osaka, N., Morishita, M., Kondo, H., and Osaka, M. (2006). Decreased activation of anterior cingulate cortex in the working memory of the elderly. Neuroreport 17, 1479-1482.

Park, D. C., Polk, T. A., Mikels, J. A., Taylor, S. F., and Marshuetz, C. M.
(2001). Cerebral aging: integration of brain and behavioral models of cognitive function. Dialogues Clin. Neurosci. 3, 151-165.

Park, D. C., Polk, T. A., Park, R., Minear, M., Savage, A., and Smith, M. R. (2004). Aging reduces neural specialization in ventral visual cortex. Proc. Natl. Acad. Sci. U.S.A. 101, 13091-13095.

Park, D. C., and Reuter-Lorenz, P. (2009). The adaptive brain: aging and neurocognitive scaffolding. Annu. Rev. Psychol. 60, 173-196.

Pattyn, N., Neyt, X., Henderickx, D., and Soetens, E. (2008). Psychophysiological investigation of vigilance decrement: boredom or cognitive fatigue? Physiol. Behav. 93, 369-378.

Paxton, J. L., Barch, D. M., Racine, C. A., and Braver, T. S. (2008). Cognitive control, goal maintenance, and prefrontal function in healthy aging. Cereb. Cortex 18, 1010-1028.

Persson, J., Sylvester, C. Y., Nelson, J. K., Welsh, K. M., Jonides, J., and Reuter-Lorenz, P. A. (2004). Selection requirements during verb generation: differential recruitment in older and younger adults. Neuroimage 23, 1382-1390.

Posner, M. I. (1980). Orienting of attention. Q. J. Exp. Psychol. 32, 3-25.

Posner, M. I., and Petersen, S. E. (1990). The attention system of the human brain. Annu. Rev. Neurosci. $13,25-42$.

Reuter-Lorenz, P. A., and Cappell, K. A. (2008). Neurocognitive aging and the compensation hypothesis. Curr. Dir. Psychol. Sci. 17, 177-182.
Reuter-Lorenz, P. A., and Lustig, C. (2005). Brain aging: reorganizing discoveries about the aging mind. Curr. Opin. Neurobiol. 15, 245-251.

Rorden, C., and Brett, M. (2000). Stereotaxic display of brain lesions. Behav. Neurol. 12, 191-200.

Rush, B. K., Barch, D. M., and Braver, T. S. (2006). Accounting for cognitive aging: context processing, inhibition or processing speed? Neuropsychol. Dev. Cogn. B Aging Neuropsychol. Cogn. 13, 588-610.

Rypma, B., Prabhakaran, V., Desmond J. E., and Gabrieli, J. D. (2001). Age differences in prefrontal cortical activity in working memory. Psychol. Aging 16, 371-384.

Solbakk, A. K., Fuhrmann Alpert, G., Furst, A. J., Hale, L. A., Oga, T. Chetty, S., Pickard, N., and Knight, R. T. (2008). Altered prefrontal function with aging: insights into age-associated performance decline. Brain Res. 1232, 30-47.

Thomsen, T., Specht, K., Rimol, L. M., Hammar, A., Nyttingnes, J., Ersland, L., and Hugdahl, K. (2004). Brain localization of attentional control in different age groups by combining functional and structural MRI. Neuroimage 22, 912-919.

Verhaeghen, P. (2011). Aging and executive control: reports of a demise greatly exaggerated. Curr. Dir. Psychol. Sci. 20, 174-180.

Wetheril, G. B., Chen, H., and Vasudeva, R. B. (1966). Sequential estimation of quantal response curves - a new method of estimation. Biometrika 53, 439-454.
Yan, P. S., and Li, C. S. R. (2009) Decreased amygdala activation during risk taking in non-dependent habitual alcohol users: a preliminary fmri study of the stop signal task. Am. J. Drug Alcohol Abuse 35, 284-289.

Zhang, S., and Li, C. S. (2012). Functional networks for cognitive control in a stop signal task: independent component analysis. Hum. Brain Mapp. 33, 89-104.

Zysset, S., Schroeter, M. L., Neumann, J., and von Cramon, D. Y. (2007) Stroop interference, hemodynamic response and aging: an event-related fMRI study. Neurobiol. Aging 28, 937-946.

Conflict of Interest Statement: The authors declare that the research was conducted in the absence of any commercial or financial relationships that could be construed as a potential conflict of interest.

Received: 26 March 2012; accepted: 07 April 2012; published online: 24 April 2012.

Citation: $\mathrm{Hu}$ S, Chao HH-A, Winkler $A D$ and $L i C R$ (2012) The effects of age on cerebral activations: internally versus externally driven processes. Front. Ag. Neurosci. 4:4. doi: 10.3389/fnagi. 2012.00004

Copyright (c) $2012 \mathrm{Hu}$, Chao, Winkler and Li. This is an open-access article distributed under the terms of the Creative Commons Attribution Non Commercial License, which permits non-commercial use, distribution, and reproduction in other forums, provided the original authors and source are credited. 